\title{
3 Research Suare \\ False-Negative Frozen Section of Sentinel Nodes in Early Breast Cancer Patients
}

\author{
Zhu-Jun Loh \\ National Cheng Kung University Hospital \\ Kuo-Ting Lee \\ National Cheng Kung University Hospital \\ Ya-Ping Chen \\ National Cheng Kung University Hospital \\ Yao-Lung Kuo \\ National Cheng Kung University \\ Wei-Pang Chung \\ National Cheng Kung University Hospital \\ Ya-Ting Hsu \\ National Cheng Kung University Hospital \\ Chien-Chang Huang \\ National Cheng Kung University Hospital \\ Hui-Ping Hsu ( $\nabla$ hphsu@mail.ncku.edu.tw) \\ National Cheng Kung University Hospital https://orcid.org/0000-0002-3285-5543
}

\section{Research}

Keywords: Early breast cancer, sentinel lymph node biopsy, false-negative frozen section, sentinel nodes, axillary lymph node dissection

Posted Date: March 15th, 2021

DOl: https://doi.org/10.21203/rs.3.rs-307059/v1

License: (c) (1) This work is licensed under a Creative Commons Attribution 4.0 International License.

Read Full License 


\section{Abstract}

Background: Sentinel lymph node biopsy (SLNB) is the standard approach of the axillary region for early breast cancer patients with clinically negative nodes. The present study investigated patients with falsenegative sentinel nodes of intraoperative frozen section (FNSNs) in real-world data.

Methods: A case-control study with a 1:3 ratio was conducted. FNSN was diagnosed when sentinel nodes (SNs) are negative in frozen sections but positive for metastasis in formalin-fixed paraffinembedded (FFPE) sections. The control was defined as having no metastasis of SNs in both frozen and FFPE sections.

Results: A total of 20 FNSN cases and 60 matched controls were enrolled from 333 SLNB patients between April 1, 2005, and November 31, 2009. The demographics and intrinsic subtypes of breast cancer were similar between FNSN and controls. The FNSN patients had larger tumor sizes in preoperative mammography $(P=0.033)$ and more lymphatic tumor emboli in core biopsy $(P<0.001)$. Four FNSN patients had metastasis in the non-relevant SNs. Another 16 FNSN patients had benign lymphoid hyperplasia of SNs in frozen sections and metastasis in the same SNs from the FFPE sections. Micrometastasis was detected in seven of 16 patients, and metastases in non-relevant SNs were recognized in two patients. All FNSN patients received a second operation with axillary lymph node dissection (ALND). After a median follow-up of 143 months, no FNSN patients developed recurrence of breast cancer. The disease-free survival, disease-specific survival, and overall survival in FNSN were not inferior to the controls.

Conclusions: The patients with a larger tumor size and more lymphatic tumor emboli have a higher incidence of FNSN. However, outcomes of FNSN patients after completing ALND were noninferior to those without metastasis in SNs. ALND provides a correct diagnosis of patients with metastasis in nonsentinel axillary lymph nodes.

\section{Background}

In Taiwan, breast cancer is the most common malignancy in women, with the incidence reaching a plateau at around 50 years of age and the prevalence increasing in all age groups in recent years $(1,2)$. With the increase in the prevalence of lymphatic spread of breast cancer, standardized operation with radical mastectomy and axillary lymph node dissection (ALND) was established by Dr. Halsted in 1894 (3). With the development of adjuvant therapy, numerous patients survive after radical resection. However, these survivors suffer from severe lymphedema over their ipsilateral upper limb after ALND (4). The technique of sentinel lymph node biopsy (SLNB) was developed to eliminate the number of resected lymph nodes in patients with clinically negative nodes (5). The clinical trial, ACOSOG Z0011, was conducted in 889 patients with cT1-2N0 breast cancers and one to two positive sentinel nodes (SNs) treated by breast-conserving therapy (BCT) with SLNB or ALND. The results revealed no difference in nodal recurrence, axillary failure, and patient survival. ALND can be avoided in early breast cancer 
patients with one to two positive SNs receiving BCT and replaced by adjuvant whole-breast irradiation (6, 7). The ACOSOG Z0011 trial did not enroll patients with large tumors or those who received a total mastectomy. Furthermore, the indication of ALND in patients undergoing total mastectomy should be considered.

The sensitivity and specificity of SLNB are the first consideration. Localization of SNs by two kinds of indicators is standard procedure. However, the intraoperative evaluation of SNs is diverse. Some institutes use cytological analysis of touch imprint, and others accept a frozen section of $\operatorname{SN}(8,9)$. Intraoperative evaluation of SNs detects metastasis and the operation changes to ALND immediately. The intraoperative frozen section of SNs has a sensitivity of $87 \%$ and a specificity of $100 \%$ (9). A $3 \%$ of patients are recalled for ALND because of a false-negative result of the frozen section during the first operation (9). The number of SNs is also a confounding factor of false-negative results of SLNB, and harvesting only one $\mathrm{SN}$ contributes to a higher rate of axillary recurrence (10). Otherwise, long-term clinical outcomes of patients with false-negative SNs are the second consideration. For patients who have micrometastasis (metastatic size greater than $0.2 \mathrm{~mm}$ and equal or less than $2 \mathrm{~mm}$ ) in SNs, the hazard ratio of regional recurrence is 4.39 for those undergoing only SLNB compared with those receiving ALND (11). Some clinical studies with several patients concluded that the incidence of regional recurrence is very low in breast cancer patients with micrometastasis in SNs $(12,13)$. For patients who have macrometastasis (metastatic size greater than $2 \mathrm{~mm}$ ) in SNs, avoiding ALND will result in the underestimation of lymph node status and may mislead the stage of cancer (14). In the present study, we investigated the predisposing factors of false-negative frozen section of SNs in patients with clinically negative nodes with breast cancer. We randomly enrolled patients planning to receive partial or total mastectomy from real-world data. This study also aimed to evaluate the long-term outcome of patients with false-negative frozen section of SNs.

\section{Patients And Methods}

The present study was designed as a case-control study with a case-to-control ratio of 1:3. The patients were diagnosed with invasive ductal carcinoma of the breast and recruited between April 1, 2005, and November 31, 2009. All these patients had clinically negative axillary or internal mammary lymph nodes. The surgical planning included SLNB. The operative method for primary breast cancer was decided by the attending surgeon and the patients after thorough discussion. The patients with ductal or lobular carcinoma in situ, metastatic disease, clinically node-positive disease, inflammatory breast cancer, other variants of carcinoma (lobular, mucinous, metaplastic, phyllodes, sarcoma, or lymphoma) and who had received neoadjuvant chemotherapy, previous axillary surgery, or radiation were excluded. The pathological stage was classified according to the criteria defined by the American Joint Committee on Cancer (AJCC) Staging Manual, 7th edition. The study was reviewed and monitored by the Institutional Review Board of National Cheng Kung University Hospital (A-ER-105-233). The intrinsic subtypes were defined by immunohistochemistry staining of estrogen receptor (ER), progesterone receptor (PR), and human epidermal growth factor type II receptor (HER2). 


\section{Identification of sentinel nodes}

All clinical information were obtained by retrospective chart review. Dual methods were used to identify SNs. All patients received a peritumoral subcutaneous injection of $1 \mathrm{mCi}$ Technetium-99 m phytate (Fujifilm RI Pharma, Chiba, Japan), after which a series of lymphoscintigraphy was performed. The SNs with radioactive signals were identified by a handheld probe of the Navigator system (RMD, Watertown, Massachusetts, USA) during operation. After anesthesia, $2 \mathrm{~mL}$ of methyl blue was injected around the tumor in the subcutaneous layer. Gentle massage for 5 min was performed from the tumor to the ipsilateral axillary region to facilitate the transmission of methyl blue along lymphatic ducts. Relevant SNs were defined as blue-stained nodes with ex vivo radioactive counts of at least $10 \%$ in situ counts. If a node was either blue-stained or had high radioactive counts, it was defined as non-relevant SNs. The number of relevant SNs sent for the frozen section was decided by the attending surgeon.

Intraoperative frozen sections were standardized. SNs of more than $4 \mathrm{~mm}$ were bisected along their long axis. Half of the nodes were embedded in Optimal Cutting Temperature Compound (OCT) (Sakura Finetec, Torrance, CA, USA) and frozen in liquid nitrogen. Small sentinel lymph nodes (less than or equal to $4 \mathrm{~mm}$ ) were completely frozen. Sections of $4 \mathrm{~mm}$ were cut and stained with hematoxylin and eosin (H\&E) for frozen examination. After preliminary frozen diagnosis, the OCT-embedded tissue of all nodes and the other half of large nodes were fixed in formalin. Three levels of permanent sections were taken on the formalin-fixed paraffin-embedded (FFPE) blocks. The final diagnosis of sentinel lymph nodes was based on the permanent sections.

\section{Enrollment of patients}

Case subjects (false-negative frozen section of sentinel node [FNSN] group) included patients who were diagnosed as negative for lymph node metastasis in frozen examination and turned out to be positive for metastasis in permanent sections from FFPE samples. Potential case subjects were identified from the registry list at the Cancer Center in National Cheng Kung University Hospital. Two chart reviewers examined the registry information. Three control subjects per case subject were selected from the same registry list and matched with the case subjects according to age of diagnosis (within 2 years), date of operation (within 1 year), tumor stage, and intrinsic subtypes (ER/PR/HER2).

All patients received preoperative examinations with sonography or mammography. The characteristics of breast tumor were recorded, and the report was categorized according to the Breast Imaging Reporting and Data System (BI-RADS) (15). Preoperative diagnosis of breast cancer was made by the cytological study of fine-needle aspiration (FNA) or pathological examination of core needle biopsy (CNB) in breast tumor. Some patients received preoperative examinations in other hospitals resulting in missing data.

\section{Statistical analysis}

The difference between the case and control groups were compared using a Chi-square test or Fisher's exact test for categorical variables. The continuous variables were analyzed by the non-parametric Mann-Whitney test. A multivariate analysis using the logistic regression model was used to determine 
the significant predictor for FNSN. Each model included age as a covariate, and the results were expressed as odds ratio (OR) with a $95 \%$ confidence interval (Cl). Survival curves were drawn by the Kaplan-Meier method, and group difference in survival time was calculated by a log-rank test. The definition of breast cancer-related disease-free survival (DFS) was the time from the date of the first operation to the date of the first recurrence of breast cancer or death from any other cause. The patients with breast cancer recurrence were defined as events, and those who died because of any other cause were defined as censored. Overall survival (OS) was the time from the date of the first operation to the date of death from any cause. Breast cancer-specific survival (BCSS) was the time from the date of the first operation to the date of death from breast cancer. Patients who died because of other causes were defined as censored while calculating BCSS. The Cox proportional hazard model for survival was applied for hazard ratio (HR) and $95 \% \mathrm{Cl}$. All statistical tests were conducted by SPSS version 17.0 (IBM, SPSS Inc., Chicago, Illinois, USA), and a $P$ value of less than 0.05 was defined as statistical significance.

\section{Results}

\section{Patient characteristics}

Between April 1, 2005, and November 31, 2009, a total of 1,810 patients were diagnosed with breast cancer in our hospital. There were 1,525 patients who received surgical intervention, whereas 333 subjects underwent SLNB. There were 20 patients with invasive ductal carcinoma and false-negative results of frozen section for SLNB, defined as the FNSN group. Matched controls were selected in those with invasive ductal carcinoma and undergoing SLNB with true-negative results from the frozen section. The demographics, tumor characteristics, and operative procedures were similar between FNSN and controls (Table 1). 
Table 1

Demographic and tumor characteristics of the subjects with false-negative frozen section of sentinel nodes (FNSN) and the controls.

\begin{tabular}{|c|c|c|c|}
\hline & $\begin{array}{l}\text { Control } \\
(n=60)\end{array}$ & $\begin{array}{l}\text { FNSN } \\
(n=20)\end{array}$ & $P$ value \\
\hline Age & $48(35-73)$ & $48(37-71)$ & 0.772 \\
\hline Operative method for breast cancer & & & 0.599 \\
\hline Total mastectomy & $22(37 \%)$ & $9(45 \%)$ & \\
\hline Partial mastectomy & $38(63 \%)$ & $11(55 \%)$ & \\
\hline Tumor size & $1.7(0.3-3.2)$ & $1.8(1.0-4.5)$ & 0.331 \\
\hline Tumor stage & & & $>0.999$ \\
\hline T1 & $43(72 \%)$ & $14(70 \%)$ & \\
\hline T2 & $17(28 \%)$ & $6(30 \%)$ & \\
\hline Nuclear grade & & & 0.418 \\
\hline Grade I & $18(30 \%)$ & $3(15 \%)$ & \\
\hline Grade II & $20(33 \%)$ & $8(40 \%)$ & \\
\hline Grade III & $22(37 \%)$ & $9(45 \%)$ & \\
\hline Extensive intraductal component & $21(35 \%)$ & $5(25 \%)$ & 0.582 \\
\hline Fascia invasion & 0 & $2(10 \%)$ & 0.060 \\
\hline Skin invasion & 0 & $1(5 \%)$ & 0.250 \\
\hline Nipple invasion & 0 & $2(20 \%)$ & 0.103 \\
\hline Estrogen receptor & & & 0.750 \\
\hline Negative & $12(20 \%)$ & $3(15 \%)$ & \\
\hline Positive & $48(80 \%)$ & $17(85 \%)$ & \\
\hline Progesterone receptor & & & 0.433 \\
\hline Negative & $25(42 \%)$ & $6(30 \%)$ & \\
\hline Positive & 35 (58\%) & $14(70 \%)$ & \\
\hline HER2/Neu & & & $>0.999$ \\
\hline Negative & 52 (87\%) & 18 (90\%) & \\
\hline Positive & $8(13 \%)$ & $2(10 \%)$ & \\
\hline
\end{tabular}




\begin{tabular}{|c|c|c|c|}
\hline & $\begin{array}{l}\text { Control } \\
(n=60)\end{array}$ & $\begin{array}{l}\text { FNSN } \\
(n=20)\end{array}$ & $P$ value \\
\hline \multicolumn{3}{|l|}{ Intrinsic subtypes } & 0.761 \\
\hline HmR-positive, HER2-negative & $43(72 \%)$ & $16(80 \%)$ & \\
\hline HER2-enriched & $8(13 \%)$ & $2(10 \%)$ & \\
\hline TNBC & $9(15 \%)$ & $2(10 \%)$ & \\
\hline
\end{tabular}

\section{Preoperative assessment}

There were 61 patients who received sonography and 78 patients who obtained a diagnostic mammography in our hospital. Sonography failed to detect the tumor in one patient in the controls. Tumor characteristics and sizes of primary breast cancer were similar between FNSN and the controls (Fig. 1A,B). The results of sonography were not different between FNSN and the controls (Fig. 1C). The FNSN patients had a larger size of breast tumors in mammography (Fig. 1D, $P=0.033$ ). Diagnostic mammography was unable to detect abnormalities in $15 \%$ of FNSN and $16 \%$ of the controls (Fig. 1E). The categorical results of BI-RADS were similar in these two groups (Fig. 1F).

There were 36 patients in the control group and 13 in FNSN who received FNA of the primary breast tumor. Three patients (two in the control and one in FNSN) in the present study only received FNA preoperatively (Fig. 1G). The gold standard for the preoperative diagnosis for breast cancer is the pathological examination of $\mathrm{CNB}$, which 77 patients received preoperatively. A total of 42 patients in the control group and 13 in FNSN received CNB in our hospital. The accumulative size of samples in CNB was equal between the two groups (Fig. $1 \mathrm{H}$ ). Three patients in the control and one in FNSN were diagnosed with ductal carcinoma in situ with microinvasion in CNB. Two patients in the control group were diagnosed with invasive carcinoma without specific types, and three had other features of carcinomas. All these patients were confirmed to have invasive ductal carcinoma in the final pathological examinations (Fig. 11).

\section{Frozen section of sentinel nodes}

All these patients received localization of sentinel lymph nodes by dual methods. Relevant SNs were sent for the frozen section. Other SNs were preserved in FFPE blocks and included in the final reports. Frozen sections of relevant SNs from four patients in FNSN were negative for malignancy in the final pathological report of FFPE blocks; however, metastatic foci were detected in the non-relevant SNs. Sixteen other patients in FNSN had results of benign lymphoid hyperplasia in the frozen section of SNs, whereas the final pathological report detected metastatic carcinomas in the FFPE blocks of SNs (Table 2). The correct evaluation of the pathological stage was performed after the second operation with ALND in FNSN patients. The patients in FNSN had a trend of a greater number of total SNs during the first operation ( $P=0.072$, Table 2 ). The number of resected and positive lymph nodes was higher in FNSN than in the control as all the FNSN patients received a second operation with ALND. The patients in FNSN 
also had a higher proportion of lymphatic tumor emboli (LTE) in CNB and an advanced nodal or AJCC tumor-node-metastasis stage in final reports (Table 2). Multivariate analysis with age, tumor size in mammography, LTE, and number of total SNs was conducted. Only LTE showed a significant predictive power for FNSN (Table 3). 
Table 2

Pathologic nodal findings from intraoperative and final diagnosis of the subjects with false-negative frozen section of sentinel nodes (FNSN) and the controls.

\begin{tabular}{|c|c|c|c|}
\hline & $\begin{array}{l}\text { Control } \\
(n=60)\end{array}$ & $\begin{array}{l}\text { FNSN } \\
(n=20)\end{array}$ & $\begin{array}{l}P \\
\text { value }\end{array}$ \\
\hline Frozen section of lymph nodes & & & \multirow{10}{*}{$<.001$} \\
\hline $\begin{array}{l}\text { Frozen section for relevant SNs and metastasis in non- } \\
\text { relevant SNs }\end{array}$ & 0 & $4(20 \%)$ & \\
\hline False-negative results & 0 & $16(80 \%)$ & \\
\hline Micrometastasis in SNs & & 7 & \\
\hline Metastasis in SNs and non-sentinel ALNs & & 1 & \\
\hline Metastasis only in SNs & & 6 & \\
\hline Macrometastasis in SNs & & 9 & \\
\hline Metastasis in SNs and non-sentinel ALNs & & 1 & \\
\hline Metastasis only in SNs & & 8 & \\
\hline True-negative results & $\begin{array}{l}60 \\
(100 \%)\end{array}$ & 0 & \\
\hline Lymphatic tumor emboli & $10(17 \%)$ & $18(90 \%)$ & $<.001$ \\
\hline Number of relevant SNs for frozen section & $1(0-9)$ & $2(1-4)$ & 0.881 \\
\hline Number of hot spots in lymphoscintigraphy & $1(1-5)$ & $1(1-4)$ & 0.570 \\
\hline Total number of SNs during 1st operation & $3(1-13)$ & $\begin{array}{l}5.5(1- \\
17)\end{array}$ & 0.072 \\
\hline \multicolumn{3}{|l|}{ Lymph node invasion } & \multirow{3}{*}{$<.001$} \\
\hline Negative & $\begin{array}{l}60 \\
(100 \%)\end{array}$ & 0 & \\
\hline Positive & 0 & 20 & \\
\hline Resected lymph nodes (including SLNB and ALND) & $3(1-13)$ & $\begin{array}{l}23(9- \\
39)\end{array}$ & $<.001$ \\
\hline Positive lymph nodes (including SLNB and ALND) & 0 & $2(1-7)$ & $<.001$ \\
\hline \multicolumn{3}{|l|}{ Nodal stage } & \multirow{2}{*}{$<.001$} \\
\hline No & $\begin{array}{l}60 \\
(100 \%)\end{array}$ & 0 & \\
\hline
\end{tabular}




\begin{tabular}{|clll|}
\hline & $\begin{array}{l}\text { Control } \\
(\mathbf{n = 6 0 )}\end{array}$ & $\begin{array}{l}\text { FNSN } \\
(\mathbf{n = 2 0 )}\end{array}$ & $\begin{array}{c}P \\
\text { value }\end{array}$ \\
\hline N1 & 0 & $17(85 \%)$ & \\
\hline N2 & 0 & $3(15 \%)$ & \\
\hline AJCC TNM stage & & & 0.001 \\
\hline Stage IA & $43(72 \%)$ & 0 & \\
\hline Stage IIA & $17(28 \%)$ & $12(60 \%)$ & \\
\hline Stage IIB & 0 & $5(25 \%)$ & \\
\hline Stage IIIA & 0 & $3(15 \%)$ & \\
\hline
\end{tabular}

Table 3

Multivariate analysis of predictors for FNSN in breast cancer patients.

\begin{tabular}{|lllll|}
\hline & & HR & $95 \% \mathbf{C l}$ & $P$ value \\
\hline Age & & 1.03 & $0.943-1.129$ & 0.499 \\
\hline Lymphatic tumor emboli & Negative & 1.0 & & \\
\hline & Positive & 47.56 & $9.23-245.04$ & $<0.001$ \\
\hline
\end{tabular}

\section{Longterm survival}

The median follow-up time was 143 months for all patients with a range of 55 to 176 months. All patients received standardized adjuvant therapy designed by attending physicians. Three patients in the control group developed recurrence during follow-up, and all of them received salvage therapy with two breast cancer-related mortalities (Table 4). No patients in FNSN developed recurrence. The DFS (HR $0.031,95 \% \mathrm{Cl} 0-23401$ in Fig. 2A), BCSS (HR 0.033, 95\% Cl 0-4465 in Fig. 2B), and OS (HR 1.522, 95\% Cl $0.279-8.317$ in Fig. 2 C) were similar between FNSN and controls. 
Table 4

Disease-free survival events and number of deaths of the subjects with false-negative frozen section of sentinel nodes (FNSN) and the controls.

\begin{tabular}{|c|c|c|c|}
\hline & $\begin{array}{l}\text { Control } \\
(n=60)\end{array}$ & $\begin{array}{l}\text { FNSN } \\
(n=20)\end{array}$ & $\begin{array}{l}P \\
\text { value }\end{array}$ \\
\hline Disease-free survival events* & 54 & 18 & $>_{0.999}$ \\
\hline Breast cancer events & $3(5 \%)$ & 0 & 0.569 \\
\hline Lung & $2(3 \%)$ & 0 & $\begin{array}{l}> \\
0.999\end{array}$ \\
\hline Liver & $2(3 \%)$ & 0 & $\overrightarrow{0.999}$ \\
\hline Bone & $3(5 \%)$ & 0 & 0.569 \\
\hline Brain & $1(2 \%)$ & 0 & $\begin{array}{l}> \\
0.999\end{array}$ \\
\hline $\begin{array}{l}\text { Regional lymph nodes (Axillary and/or internal mammary } \\
\text { chains) }\end{array}$ & $1(2 \%)$ & 0 & $\overrightarrow{0.999}$ \\
\hline Distant lymph nodes & $2(3 \%)$ & 0 & $\begin{array}{l}> \\
0.999\end{array}$ \\
\hline Local recurrence & $1(2 \%)$ & 0 & $\begin{array}{l}> \\
0.999\end{array}$ \\
\hline Non-breast cancer deaths & $3(5 \%)$ & $2(10 \%)$ & 0.594 \\
\hline Other malignancy & $2(3 \%)$ & $1(5 \%)$ & $\begin{array}{l}> \\
0.999\end{array}$ \\
\hline Sepsis & $1(2 \%)$ & 0 & $\begin{array}{l}> \\
0.999\end{array}$ \\
\hline Unknown & 0 & $1(5 \%)$ & 0.250 \\
\hline Breast cancer-related deaths & $2(3 \%)$ & 0 & $>0.999$ \\
\hline
\end{tabular}

\section{Discussion}

The standard operation for patients with early breast cancer and clinically negative nodes includes total or partial mastectomy and SLNB. In the present study, we studied the FNSN of frozen sections during the first operation. A case-matched control study was designed with a one-to-three ratio. The patients with FNSN had a larger tumor size in preoperative mammography and an increased ratio of LTE in CNB. The patients with FNSN had a greater number of SNs during the first operation. LTE was the only predictive 
variable for FNSN in multivariate analysis. Metastasis was detected in non-relevant SNs of four FNSN patients and in relevant SNs from FFPE sections of 16 patients, which was not found during intraoperative frozen sections. Seven FNSN patients had micrometastasis in SNs. Two FNSN patients had metastasis in SNs and non-sentinel axillary lymph nodes (ALNs). All 20 FNSN patients received secondary operation with ALND. Long-term outcomes of patients with FNSN or controls were similar, with no difference in DFS, BCSS, and OS between the two groups. No breast cancer-associated events developed in patients with FNSN.

Some investigators advise that intraoperative assessment is reserved for patients with clinically positive nodes or those with aggressive diseases after neoadjuvant chemotherapy (16). The cost-benefit characteristic of the frozen section is one of the reasons. Other researches still recommend intraoperative assessment of SNs. For breast cancer with clinically negative nodes, intraoperative frozen section has a sensitivity of $87 \%$, a specificity of $100 \%$, and a patient recall rate of $3 \%$ (9). Risk factors of FNSN include tumor location, lymphovascular invasion, suspicious node in the preoperative study, less than three SNs, invasive lobular carcinoma, and poorly differentiated cancer $(17,18)$. In the present study, we excluded patients with invasive lobular carcinoma because of the difficulty in the diagnosis by H\&E stain in these samples. There were 20 patients with FNSN among 333 SLNB patients. The proportion of FNSN is $6.0 \%$ in our hospital. Larger tumor size in preoperative mammography and LTE in samples of CNB were associated with increased risk of FNSN (Table 2). The patients with FNSN had a greater number of total SNs during the first operation. For a patient with LTE in CNB specimen, a tumor larger than $2.5 \mathrm{~cm}$ in preoperative mammography, and a high residual radioactivity over the axillary region during operation, the patient, and family should be informed of the higher risk of FNSN. LTE was the most powerful predictor in multivariate analysis (Table 3 ). The present study was a retrospective review of real-world data, and patient populations were not selected or well-designed. The study power might be diminished because of the heterogeneity of patients/surgeons. However, we kept monitoring these patients for more than 10 years. Our data provided real-world evidence on treatment practices for patients with FNSN.

The clinical significance of underestimating nodal staging is discussed. Anderson et a/ re-evaluated FFPE tissue blocks of SNs and found that $11 \%$ of patients have undiagnosed metastases in SNs during reevaluation (19). In the present study, four patients had cancer metastasis in non-relevant SNs but were negative for metastasis in relevant SNs. Superselection of SNs as relevant or non-relevant SNs by surgeon is not necessary. All SNs identifying with either methyl blue or with radioactive compounds should be sent for frozen section.

Sixteen other FNSN patients had results of benign lymphoid hyperplasia in the frozen section of relevant SNs, whereas the final pathological report detected metastatic carcinomas in FFPE blocks of the same nodes. The proportion of micrometastasis in SNs was seven of $20 \mathrm{FNSN}$ patients. The survival rates of FNSN patients undergoing ALND and those without SNs metastasis were similar (Fig. 2). The long-term recurrence rates were also the same (Table 4). Our results were consistent with those of other studies. The breast cancer patients with micrometastasis in SNs have similar survival as others without metastasis (6). For the patients with early breast cancer and one or two SNs containing metastasis, 10 
year OS in those treating with SLNB was noninferior to ALND (7). The SLNB successfully replaces ALND in early breast cancer (20).

The present study gathered patients from 2005 to 2009, before the publication of results in the ACOSOG Z0011 trial (6). It was the reason to perform ALND for our FNSN patients. The two-step operation with delayed ALND has similar long-term morbidity but with a longer operative time $(21,22)$. The number of lymph nodes identified is slightly reduced in delayed ALND patients without clinical significance, and the risk of lymphedema is similar between delayed and immediate $\operatorname{ALND}(23,24)$. The major risk from delayed ALND for FNSN patients comes from perioperative and anesthesia-related distress, especially in elderly patients $(25,26)$. In the present study, two patients had metastasis in SNs and non-sentinel ALNs. These two patients benefited from delayed ALND with good survival. From the results of the present study and the ACOSOG Z0011 trial, delayed ALND could be held for the patients with micrometastasis in SNs and undergoing partial mastectomy. However, delayed ALND should be considered for those with macrometastasis in SNs after evaluating the risks of a secondary operation.

\section{Conclusions}

SLNB is the standard approach of the axillary region for patients with early breast cancer and a clinically negative node. We conducted a case-matched control study. The incidence of FNSN is $6 \%$ in patients undergoing SLNB. Risk factors included a larger tumor size in preoperative mammography and an increased ratio of LTE in CNB. All 20 FNSN patients received secondary operation with ALND. Our results demonstrated that there was a decreased sensitivity of intraoperative frozen section in micrometastasis of SNs. Four of $20 \mathrm{FNSN}$ patients have metastasis in non-relevant SNs, and two other patients have metastasis in non-sentinel ALNs. Long-term survival of patients with FNSN after completing ALND was noninferior to those without metastasis in SNs.

\section{Abbreviations}

AJCC TNM stage: American Joint Committee on Cancer tumor, node, metastasis stage; ALN: axillary lymph node; ALND: axillary lymph node dissection; BI-RADS: Breast imaging reporting and data system; Cl: confidence interval; FNSN: False-negative frozen section of sentinel nodes; HER2: Human epidermal growth factor receptor 2; HmR: Hormone receptor; HR: Hazard ratio; IHC: Immunohistochemistry; SN: sentinel node; SLNB: sentinel lymph node biopsy; TNBC, triple-negative breast cancer.

\section{Declarations}

\section{Acknowledgments}

The authors are thankful to all patients who participated in the study. We were blessed with support from the late superintendent, Professor Pin-wen Lin. Furthermore, we thank Professor Tsai-Wang Chang for his teaching. We also appreciate Dr. Wen-Chung Chen, Dr. Hsueh-Li Kuo, Miss Tsai-Tsu Hung, and Miss Li- 
Ling Lin for their kind support. This manuscript has been released as a pre-print at Research Square, doi: 10.21203/rs.3.rs-23149/v1 (27).

\section{Disclosure}

There are no conflicts of interest.

\section{Authors' contributions}

Conception and design: H.P.H. Patient care: K.T.L., Y.P.C. and Y.L.K. Writing first draft of manuscript: Z.J.L. and H.P.H. Editing manuscript: W.P.C. and Y.T.H. Review and revision of the manuscript: H.P.H. and C.C.H. All of the authors have read and approved the final submitted manuscript and they participated in writing and reviewing the manuscript.

\section{Funding}

The present study was supported by grants from National Cheng Kung University Hospital (grant NCKUH11002013). This research was supported in part by Higher Education Sprout Project, Ministry of Education to the Headquarters of University Advancement at National Cheng Kung University.

\section{Availability of data and materials}

The raw data of this manuscript are available upon reasonable request to the corresponding author.

\section{Ethics approval and consent to participate}

The study was reviewed and monitored by the Institutional Review Board of National Cheng Kung University Hospital (A-ER-105-233).

\section{Consent for publication}

Yes.

\section{Competing interests}

The authors declare that the research was conducted in the absence of any commercial or financial relationships that could be construed as a potential conflict of interest.

\section{References}

1. Cancer Registry Annual Report. 2016, Taiwan. Health Promotion Administration, Ministry of Health and Welfare, Taiwan. Accessed 2 Feb 2020.

2. Sung $H$, Rosenberg PS, Chen WQ, Hartman M, Lim WY, Chia KS, et al. Female breast cancer incidence among Asian and Western populations: more similar than expected. J Natl Cancer Inst. 2015;107:djv107. 
3. Ghossain A, Ghossain MA. History of mastectomy before and after Halsted. J Med Liban. 2009;57:65-71.

4. Cormier JN, Askew RL, Mungovan KS, Xing Y, Ross MI, Armer JM. Lymphedema beyond breast cancer: a systemic review of cancer-related secondary lymphedema. Cancer. 2010;116:5138-49.

5. Johnson MT, Guidroz JA, Smith BJ, Graham MM, Scott-Conner CE, Sugg SL, et al. A single institutional experience of factors affecting successful identification of sentinel lymph node in breast cancer patients. Surgery. 2009;146:671-7.

6. Morrow M, Van Zee KJ, Patil S, Petruolo O, Mamtani A, Barrio AV, et al. Axillary dissection and nodal irradiation can be avoided for most node-positive Z0011-eligible breast cancers: a prospective validation study of 793 patients. Ann Surg. 2017;266:457-62.

7. Giuliano AE, Ballman KV, McCall L, Beitsch PD, Brennan MB, Kelemen PR, et al. Effect of axillary dissection vs. no axillary dissection on 10-year overall survival among women with invasive breast cancer and sentinel node metastasis: the ACOSOG Z0011 (Alliance) randomized clinical trial. JAMA. 2017;318:918-26.

8. Ali R, Hanly AM, Naughton P, Castineira CF, Landers R, Cahill RA, et al. Intraoperative frozen section assessment of sentinel lymph nodes in the operative management of women with symptomatic breast cancer. World J Surg Oncol. 2008;6:69.

9. Lu Q, Tan EY, Ho B, Teo C, Seah MD, Chen JJ, et al. Achieving breast cancer surgery in a single setting with intraoperative frozen section analysis of the sentinel lymph node. Clin Breast Cancer. 2013;13:140-5.

10. Kim MK, Park HS, Kim JY, Kim S, Nam S, Park S, et al. The clinical implication of the number of lymph nodes harvested during sentinel lymph node biopsy and its effects on survival outcome in patients with node-negative breast cancer. Am J Surg. 2017;214:726-32.

11. Pepels MJ, de Boer M, Bult P, van Dijck JA, van Deurzen $C H$, Menke-Pluymers MB, et al. Regional recurrence in breast cancer patients with sentinel node micrometastases and isolated tumor cells. Ann Surg. 2012;255:116-21.

12. Akay $\mathrm{CL}$, Albarracin $\mathrm{C}$, Torstenson $\mathrm{T}$, et al. Factors impacting the accuracy of intra-operative evaluation of sentinel lymph nodes in breast cancer. Breast J. 2018;24:28-34.

13. Galimberti V, Cole BF, Viale G, Veronesi P, Vicini E, Intra M, et al. Axillary dissection versus no axillary dissection in patients with breast cancer and sentinel-node micrometastases (IBCSG 23 - 01): 10year follow-up of a randomised, controlled, phase 3 trial. Lancet Oncol. 2018;19:1385-93.

14. Mittendorf EA, Hunt KK, Boughey JC, Bassett R, Degnim AC, Harrell R, et al. Incorporation of sentinel lymph node metastasis size into a nomogram predicting nonsentinel lymph node involvement in breast cancer patients with a positive sentinel lymph node. Ann Surg. 2012;255:109-15.

15. American College of Radiology. ACR BI-RADS atlas: breast imaging reporting and data system, (5th ed.), Reston, Virginia. 2013.

16. van der Noordaa MEM, Vrancken Peeters MTFD, Rutgers EJT. The intraoperative assessment of sentinel nodes - Standards and controversies. Breast. 2017;34:64-9. 
17. Wei S, Bleiweiss IJ, Nagi C, Jaffer S. Characteristics of breast carcinoma cases with false-negative sentinel lymph nodes. Clin Breast Cancer. 2014;14:280-4.

18. Lee SH, Lee HM, Lee HW, Yang BS, Park JT, Ahn SG, et al. Risk factors for a false-negative result of sentinel node biopsy in patients with clinically node-negative breast cancer. Cancer Res Treat. 2018;50:625-33.

19. Andersson Y, Frisell J, Sylvan M, de Boniface J, Bergkvist L. Causes of false-negative sentinel node biopsy in patients with breast cancer. Br J Surg. 2013;100:775-83.

20. Bouquet de Jolinière J, Major A, Khomsi F, Ben Ali N, Guillou L, Feki A. The sentinel lymph node in breast cancer: problems posed by examination during surgery. A review of current literature and management. Front Surg. 2018;5:56.

21. Olson JA, McCall LM, Beitsch P, Whitworth PW, Reintgen DS, Blumencranz PW, et al. Impact of immediate versus delayed axillary node dissection on surgical outcomes in breast cancer patients with positive sentinel nodes: results from American College of Surgeons Oncology group trials Z0010 and Z0011. J Clin Oncol. 2008;26:3530-5.

22. Goyal A, Newcombe RG, Chhabra A, Mansel RE. Morbidity in breast cancer patients with sentinel node metastases undergoing delayed axillary lymph node dissection (ALND) compared with immediate ALND. Ann Surg Oncol. 2008;15:262-7.

23. Ballal H, Hunt C, Bharat C, Murray K, Kamyab R, Saunders C. Arm morbidity of axillary dissection with sentinel node biopsy versus delayed axillary dissection. ANZ J Surg. 201888:917-921. doi: 10.1111/ans.14382.

24. Damgaard OE, Jensen MB, Kroman N, Tvedskov TF. Quantifying the number of lymph nodes identified in one-stage versus two-stage axillary dissection in breast cancer. Breast. 2013;22:44-6.

25. Braghiroli KS, Braz JRC, Rocha B, El Dib R, Corrente JE, Braz MG, et al. Perioperative and anesthesiarelated cardiac arrests in geriatric patients: a systematic review using meta-regression analysis. Sci Rep. 2017;7:2622.

26. Bettelli G. Preoperative evaluation of the elderly surgical patient and anesthesia challenges in the XXI century. Aging Clin Exp Res. 2018;30:229-35.

27. Loh ZJ, Lee KT, Chen YP, et al. non-inferiority of survival in early breast cancer patients with falsenegative sentinel nodes after completing axillary lymph node dissection. Research Square. 2020. doi:10.21203/rs.3.rs-23149/v1.

\section{Figures}



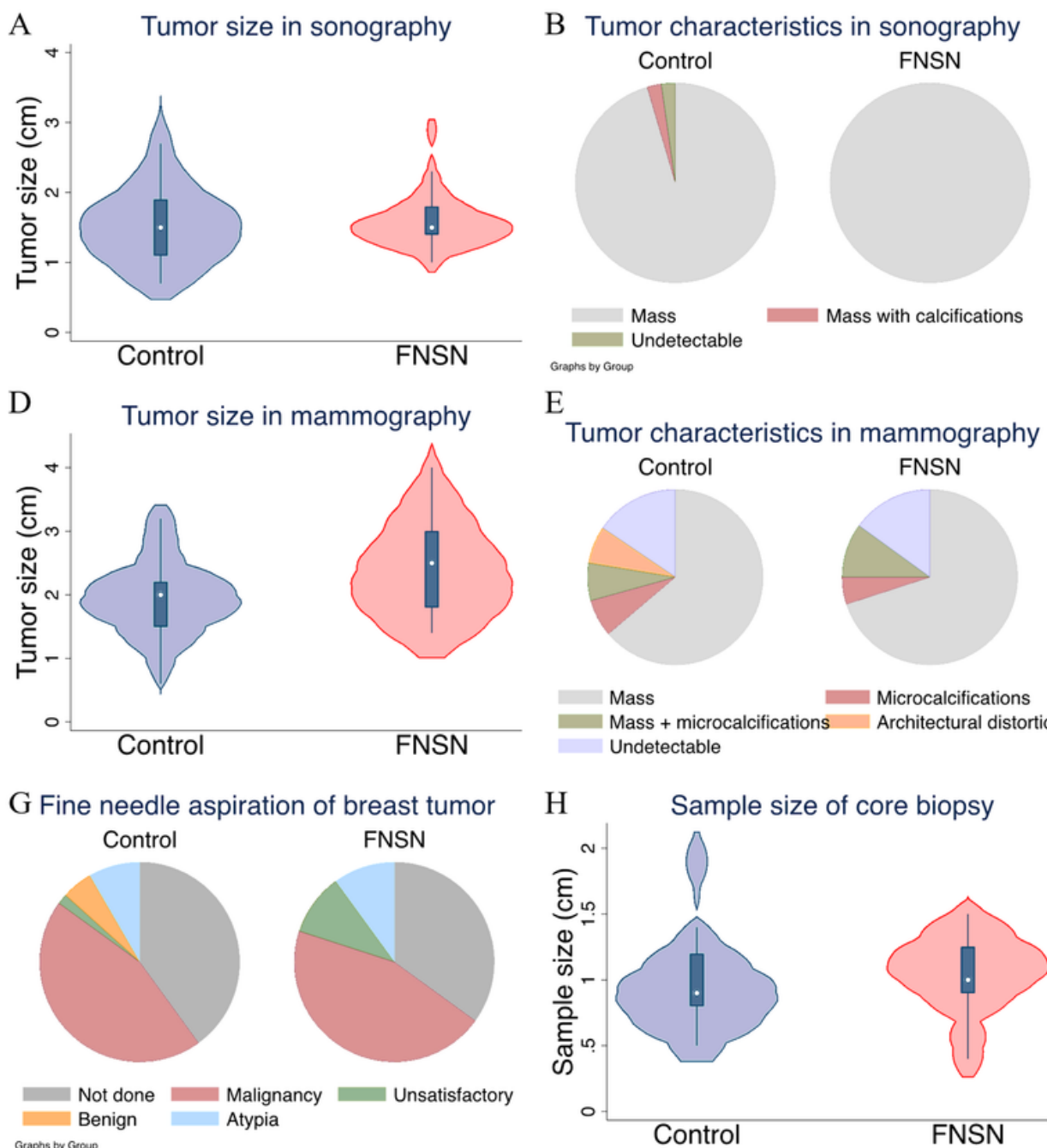

E

Tumor characteristics in mammography Control
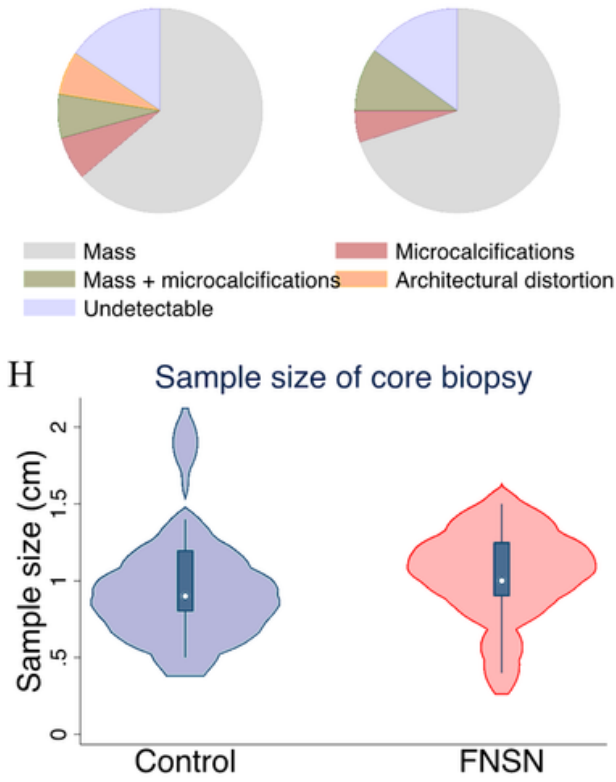

C BI-RADs category in sonography Control FNSN

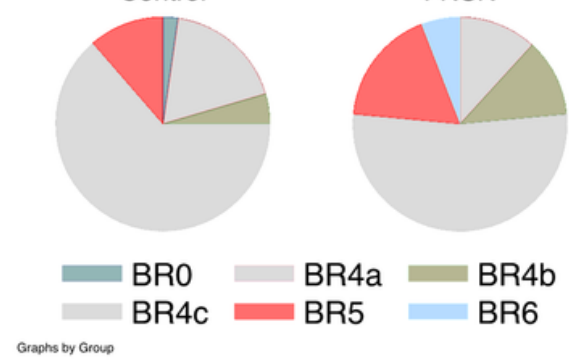

F

BI-RADs category in mammography Control FNSN

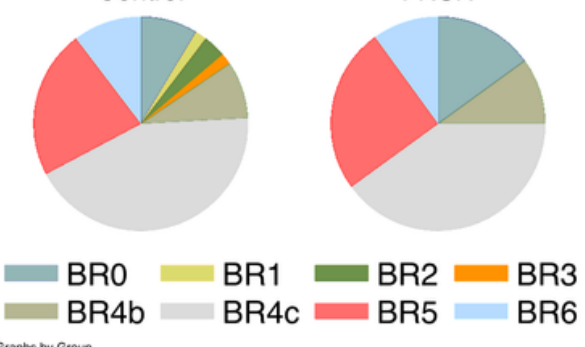

I Core biopsy of breast tumor Control FNSN

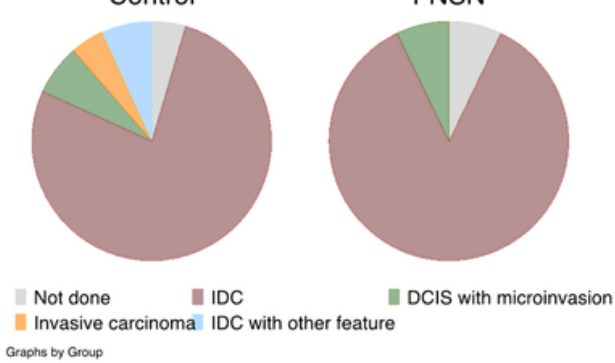

\section{Figure 1}

Preoperative imaging studies of the subjects in the present study. (A) Size of detectable tumors in sonography. (B) Tumor characteristics in sonography. (C) Results of sonography were categorized by Breast Imaging Reporting and Data System (BI-RADS). The tumor failed to be identified by sonography, and the patients who did not receive sonography in our hospital were excluded from these three analyses. (D) Size of detectable masses in mammography. The area of microcalcifications and architectural distortion was not included in the measurement of tumor size. (E) Characteristics of breast cancer in mammography. (F) Results of mammography were categorized by BI-RADS. The tumor failed to be identified by mammography, and the patients who did not receive mammography in our hospital were excluded from these three analyses. (G) Cytology results of fine-needle aspiration from a primary breast tumor. $(\mathrm{H})$ Long axis length of samples from core needle biopsy. (I) Histological results of core needle biopsy from a primary breast tumor. The patients who did not receive tissue-proved examinations in our hospital were excluded from these analyses. 

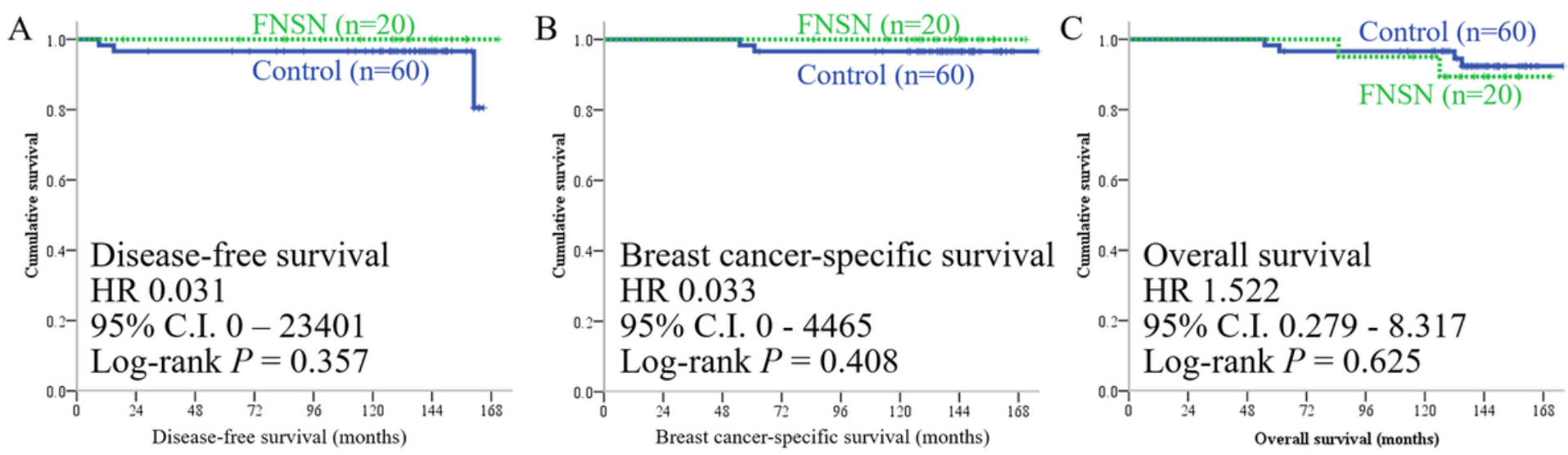

\section{Figure 2}

Kaplan-Meier survival analysis of the subjects in the present study. (A) Breast cancer-related disease-free survival, (B) breast cancer-specific survival, and (C) overall survival in the present study with a hazard ratio (HR), 95\% confidence interval $(\mathrm{Cl})$, and $\mathrm{P}$ value from log-rank test. 